\title{
A DIVISÃO DE FÍSICO QUÍMICA E OS 40 ANOS DA SBQ
}

\author{
Nelson H. Morgon ${ }^{\mathrm{a}, *}$ e Gustavo F. S. Andrade ${ }^{\mathrm{b}}$ \\ a'Departamento de Físico Química, Instituto de Química, Universidade Estadual de Campinas, CP 6154, 13083-970 Campinas - \\ SP, Brasil \\ 'Departamento de Química, Instituto de Ciências Exatas, Universidade Federal de Juiz de Fora, 36036-900 Juiz de Fora - MG, Brasil
}

Recebido em 31/03/2017; aceito em 13/06/2017

\begin{abstract}
THE PHYSICAL CHEMISTRY DIVISION AND THE FORTY YEARS OF THE BRAZILIAN CHEMICAL SOCIETY (SBQ). One of the goals of this manuscript is to celebrate the forty years of the SBQ (Sociedade Brasileira de Química). We believe using historical analysis could benefit our researchers to overcome the challenges ahead, look into the future, to the scientific progress and the way science increases its problem-solving ability. There are several topics covered by the challenges in Physical Chemistry: nanoscience and nanotechnology, single molecule studies, gas phase photochemistry and photophysics, molecular structure, spectroscopy, reaction dynamics, interfacial phenomena, energy, and so on. However, the critical challenge for our Division still is to create a desired link between experimental approaches and theoretical knowledge.
\end{abstract}

Keywords: Physical chemistry division; Forty years of the SBQ; Experimental approaches; Theoretical knowledge

\section{INTRODUÇÃO}

O pensamento científico é relativamente jovem considerando-se a presença humana no planeta. E a ciência como a conhecemos, com observação, experimentação e proposta de modelos tem $200 \mathrm{ou}$ no máximo 300 anos. No entanto, a massificação do conhecimento científico é um fenômeno muito mais recente. Após a segunda guerra mundial a humanidade experimentou e, desde então, tem experimentado, transformações sociais e tecnológicas significativas. Nenhum período da evolução humana tem sido, em termos de avanços tecnológicos, mais profícuo que os últimos 100 anos. Em parte, isso se deve ao fato de que inúmeras teorias e formalismos metodológicos terem surgido ou se consolidados no século passado.

Os países desenvolvidos, de um modo geral, tem presenciado nos últimos 70 ou 80 anos uma explosão "do fazer ciência" e suas implicações nas indústrias e tecnologias. Todavia, no Brasil e em outros países em desenvolvimento, esse fenômeno aconteceu um pouco mais tardiamente, no período que se inicia a partir da década de 1970, embora já a partir da década de 1930 o país tenha presenciado mudanças no perfil do modelo econômico de agrário-exportador para industrial. Graças ao governo Vargas, precisamente no começo da década de 1940, houve um maior incentivo industrial, com a presença marcante do Estado, criando empresas estatais relevantes, como a Companhia Vale do Rio Doce (hoje conhecida como Vale) a Companhia Siderúrgica Nacional (CSN) e a Fábrica Nacional de Álcalis (as atividades da empresa foram interrompidas em 2006), entre outras.

Para o estabelecimento de uma base industrial nacional, forte e competitiva, transformadora do perfil sócio-econômico do país, foi necessário o surgimento de recursos humanos qualificados. E dentre os vários segmentos que colaboraram para essa transformação, o setor Químico teve um papel importante e de destaque. É nesse contexto que a Química Brasileira nas últimas décadas tem mostrado ser imprescindível e possuir expressiva importância para o desenvolvimento industrial do país.

Nesse cenário ocorreu a criação da Sociedade Brasileira de Química (SBQ) em meados da década de 1970, precisamente em

*e-mail: morgon@iqm.unicamp.br
1977 “durante a Reunião Anual da SBPC, por químicos dedicados à pesquisa e ensino em universidades e institutos oficiais, liderados pelos Professores Simão Mathias, Jacques Danon e Ricardo Ferreira". ${ }^{1,2}$ Houve também contribuição essencial dos Professores Eduardo Motta Alvez Peixoto e Etelvino José Henriques Bechara, respectivamente Secretário Geral e Tesoureiro da primeira gestão da Sociedade. ${ }^{3}$ Embora tenha sido um fato marcante, sua ocorrência foi natural, face aos anseios para tal. A criação da SBQ ocorre justamente no que é conhecido como a 3a fase (de 1956 a 1989) do processo de industrialização nacional, período esse caracterizado pelo maior crescimento industrial do país em todos os tipos de indústria. Isso, associado a outro fato também muito importante que se inicia na década de 1970 e se estende pela década de 1980,,5 que foi o grande aumento do número de cursos de pós-graduação no Brasil, coloca a SBQ diante de um grande desafio, o de "assumir um papel de interlocução com as autoridades responsáveis pelas políticas de desenvolvimento científico e tecnológico do País." "1 Além disso, a SBQ passou a atuar forte e responsavelmente junto à Academia para a discussão dos acontecimentos científicos, políticos e sociais relevantes para o Brasil. E, sem dúvida alguma, ela tem desempenhado, ambas as atribuições, com responsabilidade, e além das expectativas.

\section{A Divisão de Físico Química}

São notórios os inúmeros benefícios que a ciência em geral, e a química em particular, têm fornecido à humanidade. No entanto, elas continuamente sofrem desafios que ameaçam seus sucessos contínuos. Para enfrentar tais desafios há necessidade constante de desenvolvimento de métodos e técnicas para novas aplicações ${ }^{6} \mathrm{e}$ na área de FQ e correlatas podem ser citados vários esforços nessa direção, como o desenvolvimento e/ou aplicações em vários tópicos: quantum dots; ciência e tecnologia de semicondutores; espectroscopia de single molecule ${ }^{7}$ e de sistemas complexos; nanociência e nanotecnologia, incluindo-se aí bionanotecnologia, nanoótica, nanofotônica, entre outras subáreas; interligação de dinâmica e química quântica para reações ${ }^{8}$ e dinâmica quântica para sistemas de grande escala; ${ }^{9}$ sustentabilidade e tópicos relacionados à química verde; energia e catálise, como abordagens mais gerais, e dentro dessas, energia solar e fotocatálise; técnicas espectroscópicas com alta especificidade e 
sensibilidade química em ciência forense $;^{10}$ ciência de materiais, através do desenvolvimento de dispositivos multifuncionais para conversão e armazenamento de energia ${ }^{11}$ fenômenos de interface; teoria estatística de condensação ${ }^{12}$ processos de transferências de elétrons,${ }^{13}$ redes de polímeros e o design de novos materiais $;{ }^{14}$ entre tantos outros desafios. Mas talvez o maior deles, seja o de "interligar conhecimento teórico e abordagens experimentais". ${ }^{15}$ Sendo assim, das 13 divisões que compõem a estrutura organizacional da SBQ, a Divisão de Físico Química (DivFQ) é a que mais se identifica com esse grande desafio. E deste modo, é a que tem contribuído mais destacadamente nesse sentido nos últimos 40 anos.

A DivFQ engloba as subdivisões compreendidas por pesquisas nas áreas de Físico Química (FIS), Química de Superfícies e Colóides (COL) e Química Teórica (TEO). Ela possui 990 sócios efetivos cadastrados, sendo que destes, em 2017 foram identificados 221 como os sócios ativos. Sua diretoria para o biênio 2016-2018 é composta pelos Profs. Nelson H. Morgon/UNICAMP (Diretor) e Gustavo F. S. Andrade/UFJF (Vice-Diretor). O número de sócios ativos em 2017 reverte uma tendência de número de inscritos nas Reuniões Anuais da SBQ (RASBQ) como pode ser observado na Fig. 1. Ao longo dos últimos 20 anos têm acontecido oscilações com tendência decrescente no número de participantes vinculados à DivFQ. Vários fatores podem ser atribuídos a esse comportamento, como a migração de pesquisadores para outras divisões, surgimento de inúmeros outros eventos científicos, entre outros. Apesar de ser um fator marcante, essa migração não é recente. Como notado por Santos e de Souza, já em 2002, ${ }^{16}$ no artigo sobre o desenvolvimento da área de Físico-Química no Brasil durante os primeiros 25 anos da SBQ, há presença de trabalhos relacionados à área de Físico-Química em diferentes divisões da Sociedade durante as Reuniões Anuais, como na Química de Materiais, em Química Inorgânica e em Fotoquímica. Além disso, Santos e de Souza ${ }^{16}$ realizaram um estudo qualitativo de grande relevância sobre o desenvolvimento da Físico-Química, como uma área independente de pesquisa em nosso país, inclusive diferenciando essa físico-química, que utiliza métodos e modelos fenomenológicos, da outra denominada chemical physics, que utiliza metodologia avançada e interpretações baseadas fortemente na teoria quântica. Essa, apresenta desenvolvimento menos consistente em nosso país. A opção feita pelos autores deste artigo foi discutir o desenvolvimento da DivFQ ao longo dos anos na RASBQ. Na discussão desse desenvolvimento na próxima seção, esforços foram feitos para mostrar que as mudanças ocorridas na DivFQ se correlacionam fortemente com o processo de desenvolvimento da área como um todo no Brasil e no exterior.

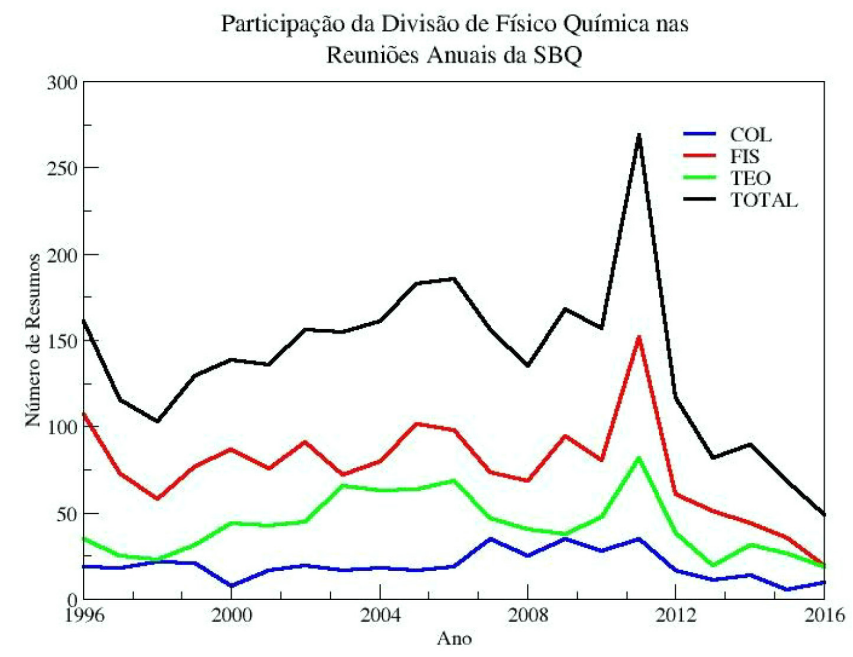

Figura 1. Número de inscritos nas Reuniões Anuais da Sociedade Brasileira de Química vinculados à Divisão de Físico Química no período de 1996 a 2016.

\section{A Divisão de Físico Química em Foco}

Na Fig. 2 tem-se a evolução do número de resumos submetidos às RASBQ nos últimos 20 anos (1996 a 2016). O ano de 2011 mostra uma grande participação de pesquisadores vinculados à Divisão. O número de resumos apresentados na 34a Reunião Anual, em Florianópolis - SC, deveu-se à comemoração do Ano Internacional da Química. Novamente observa-se um declínio nos últimos anos na submissão de resumos, cujas razões foram descritas acima, aliadas também em parte, à diminuição do financiamento à pesquisa no país.

Inscritos pela Div. de FQ nas RASBQ

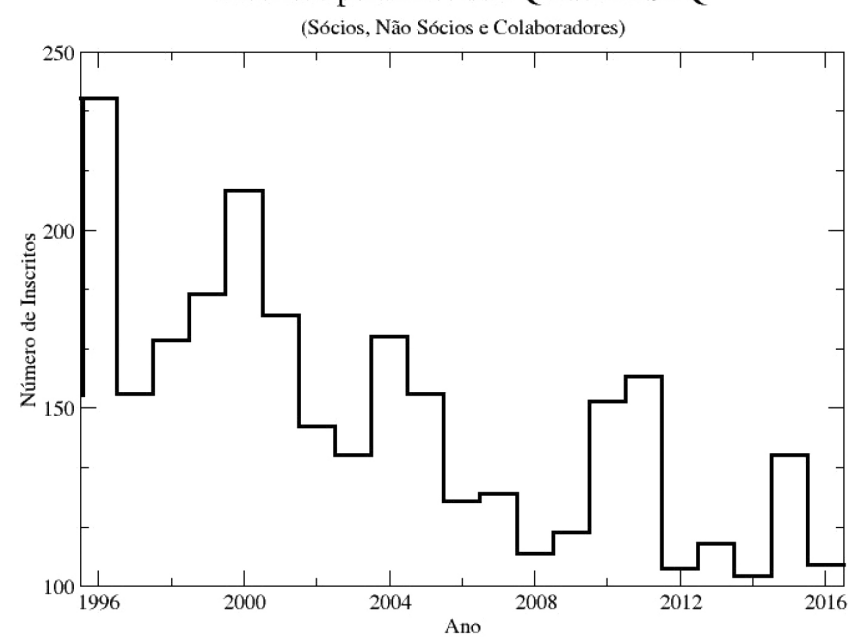

Figura 2. Número de resumos submetidos às Reuniões Anuais da Sociedade Brasileira de Química vinculados à Divisão de Físico Química no período de 1996 a 2016.

Após dez anos de um crescimento rápido, mobilizando químicos de todo o país em suas Reuniões Anuais, como parte integrante das programações das reuniões anuais da Sociedade Brasileira para o Progresso da Ciência (SBPC), pode ser observado que a DivFQ manteve a chama da qualidade em sua participação, quer como parte das reuniões anuais, inicialmente da SBPC e posteriormente nas reuniões independentes organizadas pela SBQ.

A seguir serão destacados alguns aspectos dessa participação ao longo dos últimos anos. A seleção apresentada foi feita do ponto de vista cronológico, relevante e/ou aleatório, sem preocupação com algum rigor científico na sua escolha. Deseja-se nessa discussão apresentar qualitativamente um panorama dos desenvolvimentos e das tendências da área, a partir da participação da DivFQ nas RASBQ.

Na 39a RASBPC realizada em 1987 em Brasília, DF, os tópicos em destaques dentro da DivFQ da SBQ eram "Cristais líquidos e detergentes, Eletroquímica, Espectroscopia e Cinética e Catalisadores". Foram apresentados 133 resumos em FQ, como o que trata da "Cinética de oxidação de d(-) frutose pelo vanádio (V) em meio ácido", de Takashima ${ }^{17}$ ou o de Quezado sobre "Espectro infravermelho e ligações de hidrogênio em polímeros perfluorados". ${ }^{17}$ Dos 29 resumos de Química Teórica, tem-se o de Degréve, sobre "Aglomerados iônicos em soluções de eletrólitos" 17 e o de Livotto e Takahata abordando o "Estudo do substituinte sobre a afinidade eletrônica de derivados de p-benzoquinona". ${ }^{17}$ Outro destaque nessa reunião foi a Sessão Especial sobre "Os 10 anos da SBQ", coordenada pelo Prof. Angelo. C. Pinto.

Passados mais dez anos, há sem dúvida alguma um amadurecimento da comunidade dos químicos brasileiros o que acaba refletindo nas reuniões anuais. Na 19a RA, a primeira em Poços de Caldas, MG, Prof. Marco Chaer em sua Conferência Convidada (CC) sobre 
"Modelagem Molecular: do Sonho à Realidade", apresenta os avanços que estão por vir na "utilização de MM nas áreas de catálise, corrosão, propriedades mecânicas de polímeros, enovelamento de proteínas e de fenômenos interfaciais", (CC07). Isso hoje é fato corriqueiro em diversos laboratórios de simulação computacional, e até mesmo como ferramenta de bancada. Nessa reunião já pode ser observado um fenômeno característico que é a geração de novas divisões da sociedade. Pode ser observado no programa da RA a presença das divisões de Catálise, Eletroquímica e Eletroanalítica e Fotoquímica, além da própria DivFQ; que foram, dez anos antes, grande parte da temática da DivFQ na 39aㅗ RA da SBPC, como citado anteriormente.

A Profa. Schor na 20a RA fala sobre o tema "Dinâmica Química", destacando-a como uma área vigorosa e emergente em química; por sua vez a femtoquímica, um ramo daquela, permitirá o acompanhamento de reações em tempo real (CC08). Na mesma RA, o Prof. Galembeck ministrou um curso denominado "Nanopartículas e sistemas organizados", antevendo a importância da nanociência. Atualmente, pesquisas em nanociência e nanotecnologia permeiam diversas áreas da SBQ.

A DivFQ, desde sua criação, tem tido uma preocupação constante com dois aspectos: a divulgação científica oriunda de pesquisas de qualidade, e a qualidade do ensino. Esse último tema tem sido abordado em diversos momentos ao longo desses 40 anos, quer em Sessões Coordenadas, Workshops ou em CC. Na CC07 da 21 $1^{\text {a }}$ RA proferida pelo Prof. Donald A. McQuarrie, o tema "Quanta matemática deve ser ensinada em um curso de Físico-Química?”, ilustra bem essa preocupação. McQuarrie falou sobre tópicos básicos de matemática que são corriqueiramente usados para resolver muitos problemas em cursos introdutórios de FQ e discutiu o uso de alguns métodos numéricos em várias aplicações. Além dessa demonstração da importância do ensino na área de Físico-Química, também nessa RA houve a apresentação do Prof. J. M. Riveros. Na conferência plenária "O renascimento da Química em fase gasosa: reatividade e estrutura de espécies simples até agregados moleculares”, ele descreve novas metodologias envolvendo a forte interação entre técnicas experimentais avançadas e de alta resolução e cálculos computacionais de alto nível, que em diversos aspectos define a pesquisa em Físico Química nesse início de século XXI.

"Química Computacional" foi o destaque na Conferência de Abertura (CA) da 22 aA. Na palestra, o Prof. Roy E. Bruns destaca, entre outros assuntos relevantes sobre o uso de computadores em química, que a pesquisa quimiométrica tem sido importante para o desenvolvimento de modelos empíricos em diversas áreas. Hoje se vê em muitos laboratórios de pesquisa e em indústrias a quimiometria como uma ferramenta útil no tratamento de dados.

O que de relevante aconteceu na CA da 23a RA foi sinalização de que à próxima geração de profissionais da química caberia a tarefa de implementar ações que fortalecessem a ligação Brasil-Portugal. Mas, além disso, em termos experimentais, notou-se qualitativamente que tanto a sessão coordenada como os painéis apresentados pela DivFQ trataram temas como: aspectos espectroscópicos de diversos sistemas, como efeitos de solvente e confinamento, monitorados por técnicas como a espectroscopia Raman e a apresentação da técnica de lentes térmicas para a caracterização de filmes e diâmetro de feixe de lasers. Também houve a apresentação na forma de painéis de diversos trabalhos relacionados à Química de Superfícies e Colóides.

$\mathrm{Na} 24$ a RA novamente Prof. Riveros, em sua palestra na CA, trata da questão "Estrutura e reatividade em química", e reforça a necessidade das metodologias experimentais e teóricas na caracterização de reatividade e estrutura em fase gasosa, permitindo a análise individual de moléculas. De certa maneira isso contempla parte do desafio de "interligar conhecimento teórico e abordagens experimentais". Esse aspecto foi também abordado pelo Prof. Paulo S. Santos em sua CC
"Espectroscopia Raman: Uma viagem no Espaço e no Tempo", sobre os avanços e desafios das interações computacional e experimental, além do desenvolvimento instrumental da técnica no Brasil.

Na 25 RASBQ, na esteira da comemoração dos 25 anos da entidade, a palestra de Chaer novamente tratou de desenvolvimentos recentes na conceituação de estrutura e ligação química, pontuando perspectivas para a área; já Tada e El Seoud apresentaram trabalho na sessão de pôsteres de Química de Superfícies e Colóides sobre o "Solvatocromismo em agregados micelares demonstrando o efeito da transição de micela esférica para cilíndrica", (QC-011). Silva e de Torresi apresentaram a aplicação de NOESY RMN e XPS, na caratecrização de processos envolvendo polímeros em solução.

Sem dúvida alguma os estudos sobre nanomateriais de carbono (NC) tornaram-se relevantes nos últimos anos. Assim, em sintonia com avanços tecnológicos recentes o tema foi tratado na CC05 da 26aㅡ. RA. Pimenta em sua conferência sobre "Nanotubos de Carbono" enfatizou que a espectroscopia Raman é uma ferramenta bastante útil no estudo de propriedades estruturais e eletrônicas de tais sistemas, bem como descreveu aplicações que tem despertado o interesse da comunidade científica desde então.

A questão energética não foi deixada de fora das discussões da Divisão. Na 27a RA “A Química e a físico química na produção e no uso do biodiesel", foi abordada considerando-se o desenvolvimento do conhecimento da cadeia produtiva e de utilização de combustíveis alternativos. O tema foi tratado na palestra proferida por Prof. Miguel J. Dabdoub Paz. Nessa RA houve a presença de pesquisadores de outros países da América Latina, uma vez que se tratava de um congresso em conjunto com o XXVI Congreso Latinoamericano de Química.

Prof. Samios na 28 ${ }^{\mathrm{a}}$ RA apresenta em sua conferência (CC09) “A evolução da Química: da simplicidade à complexidade", traçando uma visão evolutiva da química em relação à sociedade. Pode-se destacar também a CA proferida por Dupont, que versou sobre estrutura e propriedades de líquidos iônicos, um tema que vinha interessando à comunidade tanto nas abordagens teóricas quanto nos aspectos experimentais já havia algum tempo. No Workshop da DivFQ versando sobre "Técnicas Avançadas em Espectroscopia usando a Luz Síncrotron", foi novamente possível a divulgação das enormes potencialidades da radiação Síncrotron na solução de problemas químicos.

Dois destaques podem ser citados na participação da DivFQv na 29a RA. O Workshop "Boltzmann e a química moderna", sob a coordenação do Prof. L. C. G. Freitas e na Sessão Coordenada, a apresentação de C. A. Silvério da "Proposta de modelo cinético para a reação foto-fenton na presença de íons cloreto".

Os Workshops da Divisão passaram a ter cada vez maior destaque nas reuniões anuais. Na 30를 RA sob a coordenação do Prof. M. E. Zaniquelli, o tema "Aspectos Dinâmicos da Físico Química" foi discutido por um bom número de participantes.

As Sessões Coordenadas (SC) também têm tido um destaque maior nos últimos tempos. Nas SC da DivFQ tem se procurado envolver cada vez mais os jovens pesquisadores, principalmente pós-graduandos e pós-doutores. Tem-se também realizado esforços para divulgar trabalhos de Iniciação Científica, já vislumbrando o futuro da Divisão. Na 31ª RA, na SC de FIS/TEO, mesclaram-se apresentações de trabalhos experimentais, como o F. C. de Oliveira sobre o "Estudo da aniquilação de pósitron em misturas mecânicas e soluções sólidas formadas por $\mathrm{Tb}(\mathrm{dpm})_{3}$ e $\mathrm{Eu}(\mathrm{dpm})_{3}$ ", e trabalhos teóricos, exemplificado pelo apresentado por L. H. K. Queiroz Jr. chamado "Cálculos teóricos de $\delta$ e $J$ do epóxido de $\alpha$-pireno: atribuição inequívoca dos sinais de RMN e da estereoquímica relativa". O Workshop da DivFQ nessa RA foi dedicado ao tema "Interações entre moléculas e partículas: implicações em biofísica e materiais", com apresentações sobre a temática por pesquisadores de destaque no cenário nacional. 
Prof. J. Hofkens, na 32a RA, em sua apresentação sobre espectroscopia de molécula única (single molecule spectroscopy - SMS) relatou que a técnica tem se estabelecido como uma nova ferramenta especialmente útil para estudar sistemas biológicos, que por sua natureza altamente heterogênea são alvos perfeitos para SMS. Na SC, a apresentação de dos Santos sobre "Espectroscopia Raman Intensificada pela superfície no regime de uma única molécula", tratou da técnica de espectroscopia vibracional para single molecule.

Na 33 ${ }^{\text {a }}$ RA ocorreram dois Workshops patrocinados pela Divisão de Físico Química. Um que celebrou as conquistas científicas do Prof. José Manuel Riveros, por ocasião do seu 70ํaniversário, sob a coordenação do Prof. Sérgio Galembeck. E outro sobre os "Avanços e Perspectivas em Físico Química de Colóides e Superfícies", coordenado pelo Prof. Fernando Galembeck. Além do Workshop, a "Ciência de Superfícies e Colóides", tiveram destaques a CC de Loh, "A relação entre termodinâmica e a estrutura de agregados de surfactantes: quem controla quem?", e a Sessão Temática coordenada por Sabadini "Interfaces: onde teoria e experimento se encontram", onde foram discutidos aspectos de técnicas avançadas em conjunção com métodos teóricos para o estudo desses sistemas.

Além do caráter educativo assinalado em vários momentos pela Divisão ao longo desses 40 anos, outra preocupação de caráter formador foi o de apresentar às novas gerações proeminentes pesquisadores que deram contribuições fundamentais para a área. $\mathrm{Na} 34^{\mathrm{a}}$ RA foi organizado o Workshop "Grandes nomes que influenciaram a Química atual" sob a coordenação da Profa. Nadya Pesce da Silveira. As palestras apresentadas por pesquisadores de destaque da Divisão trataram das obras e da vida de nomes como: Boltzmann, Langmuir, Helmholtz, Gibbs, Ostwald, entre outros.

A questão da integração teoria e experimento tem sido um desafio constante, e considerado em vários momentos pela Divisão. Foi dado destaque tanto no minicurso "Fundamentos e aplicações de espectroscopia eletrônica nas regiões do VIS-UV-Raios-X", por Lago e Riveros, como em trabalhos selecionados para participação nas sessões coordenadas, como o apresentado por Ivania Ferreira na 35" RA, abordando a "Espectroscopia SERS e cálculos teóricos de agentes quelantes de metais para aplicação na Doença de Alzheimer".

Espectroscopia é uma área de pesquisa extremamente relevante, importante e atual dentro da FQ. Na 36ª RA o tópico foi tema do Workshop "Recentes avanços da espectroscopia na caracterização de materiais e sistemas", coordenado pelo Prof. Paulo Netz. O tema também foi destaque nas CC apresentadas por Berberan e Santos e Martinho, relacionados aos desenvolvimentos da espectroscopia de fluorescência.

Nanotecnologia tem se destacado no cenário internacional nas últimas décadas. Graças à apresentação de Daniel Ugarte na conferência "Advanced characterization of individual nanosystems by electron microscopy techniques", da 37를 conheceu-se um pouco mais das "novas propriedades físicas e químicas dos nanosistemas, que os colocam como um dos elementos mais promissores para o desenvolvimento de novas tecnologias", bem como o status e perspectivas para os métodos de caracterização por microscopia eletrônica e o ponto desafiador associado à formação de recursos humanos nesse campo

Outro tópico dentro da Divisão que tem sido lembrado sistematicamente, graças à importância que vem adquirindo nos últimos 50 anos, é o da modelagem molecular. Em duas RAs, na 38a e 39a o assunto foi apresentado. Primeiramente pelo Prof. Dominic Tildesley, presidente da Royal Society of Chemistry, que ministrou palestra sobre modelagem multiescala de interfaces (Multiscale Modelling of Interfaces), na qual ele discutiu e apresentou os desenvolvimentos que resultaram na modelagem precisa de interfaces e na previsão da tensão superficial de um líquido simples. Por outro lado, o Prof.
Munir Skaf em "Molecular modeling for lignocellulosic biomass degradation", enfatizou que as "técnicas avançadas de modelagem também desempenham papéis fundamentais na compreensão da nanoarquitetura de polissacarídeos e montagem de lignina em paredes celulares de plantas".

Também na 39a RA outro tópico importante, o das interfaces, foi abordado no Workshop "Química nas interfaces sólido/líquido e sólido/gás", sob a coordenação do Prof. N. H. Morgon, que teve apresentações versando sobre tópicos experimentais e teóricos relacionados à temática.

\section{E o que esperar da DivFQ na 40aㅗ RASBQ?}

O ano de 2017 será muito especial para a comunidade de químicos do Brasil. A 40aㅡ Reunião Anual da Sociedade Brasileira de Química será realizada conjuntamente com o $46^{\text {th }}$ World Chemistry Congress e com a IUPAC $49^{\text {th }}$ General Assembly. Grande destaque será dado à pesquisa científica brasileira na área de química, bem como aos pesquisadores do país, os quais terão a oportunidade de conhecer o que há de mais atual no cenário internacional. Uma oportunidade ímpar de fomentar e trocar ideias. E a DivFQ será atuante, discutindo, questionando, oferecendo aos associados oportunidades de conhecer grandes temas atuais da área, bem como os desafios que estarão sendo colocados para o futuro imediato ou mais além. Nesse sentido, o simpósio Physical, Biophysical and Computational Chemistry vai se dedicar à apresentação de temas atuais em (Bio)Físico Química, Química Teórica, Automontagem, Fronteiras em Espectroscopia e Química Atmosférica.

\section{CONSIDERAÇÕES FINAIS}

Com este trabalho procurou-se apresentar o desenvolvimento da DivFQ, uma das Divisões que atua na SBQ desde sua fundação, nos últimos 40 anos. A Divisão passou por diversas transformações que aconteceram naturalmente, fruto do amadurecimento da sociedade científica como um todo. A partir da DivFQ, originou-se outras divisões como: Fotoquímica, Eletroquímica e Eletroanalítica e Catálise. Procurou-se demonstrar que a DivFQ, em conjunto com essas e outras divisões, possuem a percepção de que através de seus encontros, devem promover o debate e a disseminação de tópicos, estudos e/ou ideias relevantes na ciência contemporânea. Deste modo, que com o passar dos anos, a DivFQ tem procurado fomentar discussões de tópicos que apresentem o estado-da-arte da pesquisa na área de Físico-Química, Química Teórica e de Química de Superfícies e Colóides.

\section{AGRADECIMENTOS}

Os autores gostariam de agradecer às agências financiadoras CNPq (Procs. №s: 472485/2013-0 e 306975/2013-0), FAPESP (Procs. №s: 08293-7/2013 e 22338-9/2015), FAPEMIG (CEXAPQ-01283-14) e à Diretoria da SBQ pelo convite para contribuir para o número especial de Química Nova relativo aos 40 anos da SBQ em 2017.

\section{REFERÊNCIAS}

1. Galembeck, F.;'Sobre a SBQ”, http://www.sbq.org.br/pagina/sobre-sbq, acessada em Maio de $\mathbf{2 0 1 7}$.

2. Bechara, E. J. H.; Viertler, H.; Quim. Nova 1997, 20, 63.

3. Peixoto, E. M. A.; Quim. Nova 2004, Ed. Especial, 6.

4. Pinto, A. C.; Andrade, J. B.; Vieira, P. C.; Pardini, V. L.; Quim. Nova 2004, Ed. Especial, 221. 
5. Brocksom, T. J.; Andrade, J. B.; Quim. Nova 2004, Ed. Especial, 270.

6. Oliveira, Jr., O. N. J.; Phys. Chem. C 2016, 120, 5273.

7. Kondo, T.; Chen, W. J.; Schlau-Cohen, G. S. ;Chem. Rev. 2017, 117, 860.

8. Clary, D. C.; Abstr. Pap. Am. Chem. S. 2014, 248, 303.

9. Akimov, A. V.; Prezhdo, O. V.; Chem. Rev. 2015, 115, 5797.

10. Ewing, A. V.; Kazarian, S. G.; Anal. 2017, 142, 257.

11. Huang, Y.; Zhu, M.; Huang, Y.; Pei, Z.; Li, H.; Wang, Z.; Xue, Q.; Zhi, C.; Adv. Mater. 2016, 28, 8344.

12. Ushcats, M. V.; Bulavin, L. A.; Sysoev, V. M.; Bardik, V. Y.; Alekseev, A. N.; J. of Mol. Liq. 2016, 224, 694.
13. Kaiser, R. I.; Parker, D. S. N.; Mebel, A. M.; Annu. Rev. Phys. Chem., Ed. M. A. Johnson and T. J. Martinez - Annual Review of Physical Chemistry, 2015.

14. Wang, R.; Sing, M. K.; Avery, R. K.; Souza, B. S.; Kim, M.; Olsen, B. D.; Acc. Chem. Res. 2016, 49, 2786.

15. Uskokovic, V. U. K.; Found. of Sci. 2010, 15, 303.

16. Santos, P. S.; Souza, G. G. B.; Quim. Nova, 2004, 25, 159

17. Livro de Resumos da Reunião Anual Sociedade Brasileira para o Progresso da Ciência (SBPC), 1987, p. 469, 500, 577 e 582 Universidade de Brasília - UnB, Brasília, DF. 